\title{
Vaciamiento ganglionar en carcinoma escamoso transglótico
}

\section{Ganglionic drainage in scaly transglotic carcinoma}

\author{
Juan Carlos Bravo $\mathrm{Y}^{1}$, Mariela C Torrente $\mathrm{A}^{2}$, Loreto A Nicklas $\mathrm{D}^{1}$.
}

\begin{abstract}
RESUMEN
Introducción: 日 manejo de los ganglios cervicales es fundamental en el tratamiento y pronóstico de los pacientes con carcinoma escamoso de laringe. Existe consenso en realizar vaciamiento ganglionar cervical en ausencia de adenopatías clínicas (NO) cuando el riesgo de adenopatías metastásicas ocultas supera el 20\%. laríngeo transglótico (CTG) se caracteriza por presentar una incidencia de metástasis cervicales ocultas de 30\% a 40\%. Objetivo: Evaluar los hallazgos histopatológicos en los ganglios cervicales de los pacientes portadores de CTG, analizando la incidencia de metástasis ocultas y su asociación con factores de riesgo. Material y métodos: Estudio retrospectivo mediante la revisión de las fichas clínicas de pacientes portadores de CTG, sin tratamiento previo, manejados quirúrgicamente en el Hospital San Juan de Dios entre los años 1994 y 2002. Resultados: Se evaluaron 20 pacientes, 4 (20\%) se presentaron con adenopatías clínicas, realizándose en 2 casos vaciamiento radical y en los 2 restantes disección funcional. Los pacientes No fueron 16 y se les efectuó un vaciamiento funcional bilateral. Se detectaron metástasis ocultas en 12,5\% de los pacientes NO. Conclusión: Los carcinomas de ubicación transglótica NO no se beneficiarían de un vaciamiento ganglionar cervical.
\end{abstract}

Palabras clave: Carcinoma transglótico, metástasis ganglionar oculta

\section{SUMMARY}

Introduction: The handling of the cervical ganglion is basic in the treatment and prognosis of patients with scaly carcinoma of the larynx. There is consensus on doing ganglionic cervical drainage in the absence of clinical adenopathies (NO) when the risk of hidden metastasic adenopathies is over $20 \%$. Laryngeal transglotic carcinoma (LTC) is characterized for presenting an incidence of hidden cervical metastasis of 30 to $40 \%$. Objective: To evaluate histopathological findings in the LTC patients' cervical ganglions, analyzing the incidence of hidden metastasis and their association with risk factors.

\footnotetext{
${ }^{1}$ Médico del Servicio de Oorrinolaringología, Hospital San Juan de Dios.

${ }^{2}$ Médico del Departamento Otorrinolaringología de la Facultad de Medicina de la Universidad de Chile, Hospital San Juan de Dios.
} 
Material and methods: Retrospective study through revision of the clinical files of LTC carrier patients, without previous treatment, surgically treated at the San Juan de Dios Hospital between years 1994 and 2002. Results: 20 patients were evaluated, 4 (20\%) presented clinical adenopathies, undergoing in 2 cases radical drainage and in the other 2, functional dissection. The NOpatients were 16 and a bilateral functional drainage was made. Hidden metastasis were detected in $12,5 \%$ on the NO patients. Conclusion: Carcinomas of NO transglotic location did not benefit from a cervical ganglionic drainage.

Key words: Transglotic carcinoma, hidden ganglionic metastasis.

\section{INTRODUCCION}

日 carcinoma de laringe corresponde al $1 \%-2 \%$ de las neoplasias malignas del organismo y al $25 \%$ de los carcinomas escamosos en el área de cabeza y cuello ${ }^{1}$. La variedad histológica predominante corresponde al carcinoma epidermoide en 95\%$96 \%^{2-4}$. Dependiendo de la zona anatómica comprometida por el tumor, varía la presentación clínica y el compromiso ganglionar cervical.

La presencia o el riesgo de presentar metástasis cervical es un elemento fundamental a considerar en la planificación terapéutica, siendo el factor pronóstico más importante en el manejo de esta patología La metástasis cervical determina una disminución de la sobrevida de $40 \%-50 \%{ }^{5-7}$. Además, el $50 \%$ de los fracasos del tratamiento son secundarios a recidiva regional. La presencia de metástasis cervicales varía de acuerdo al sitio y extensión del primario, presentando patrones de diseminación linfática ordenados y predecibles ${ }^{8}$. I manejo del cuello estará determinado por la presencia de adenopatías clínicamente identificables, tipo de manejo del primario y riesgo de metástasis ocultas. En aquellos pacientes sin adenopatías clínicas (NO), se discute si el manejo ganglionar debe ser inicialmente quirúrgico o es preferible esperar la aparición de metástasis para realizar un vaciamiento ganglionar. 日 manejo quirúrgico en NO proporcionaría un mejor control regional que la observación, ya que cuando las adenopatías se hacen evidentes son inoperables en 36\% $\%^{9-11}$. Se recomienda la disección sistemática de las cadenas ganglionares del cuello cuando la incidencia de metástasis oculta es mayor al $20 \%$, situación que se presenta de preferencia en localizaciones supraglótica y transglótica del carcinoma laríngeo (20\% a 40\%).

日 carcinoma transglótico (CTG) se define por el compromiso de la cuerda vocal y la banda cruzando a través del ventrículo. Su origen podría estar en la glotis o supraglotis, ascendiendo o descendiendo, respectivamente, en su extensión a través del ventrículo, o bien, a nivel del fondo del ventrículo para luego extenderse en ambas direcciones $^{12}$.

Las características histopatológicas del CTGson la extensión submucosa a través de la banda vocal, invasión del espacio paraglótico, invasión cartilaginosa y metástasis en ganglios cervicales de alrededor de $30 \%-40 \%{ }^{13}$. La particularidad de esta localización radica, además, en el compromiso de estructuras supraglóticas de rica vascula-rización linfática, poseedoras de dos sistemas linfáticos, uno de los cuales es de ubicación superficial submucoso, con una gran cantidad de vasos y anastomosis que cruzan la línea media, ubicado principalmente a nivel de banda, ventrículo y pliegue aritenoepiglótico. Esta densa vascularización linfática determina una elevada incidencia de metástasis ganglionar con cifras que varían entre $\pm 30 \%$ $40 \%$ y de metástasis oculta de $\pm 25 \% 14,15$. En su extensión, a través del espacio paraglótico, provoca un mayor compromiso del esqueleto laríngeo y mayor riesgo de infiltrar la pared medial del seno piriforme, siendo más propenso a presentar metástasis bilateral ${ }^{16}$. 
Myers en el año $1999^{17}$ analizó la incidencia de metástasis oculta en ganglios cervicales en diferentes localizaciones de carcinoma laríngeo, describiendo en el CTG 11\%-12\%, situación que haría cuestionable el vaciamiento ganglionar cervical electivo en pacientes NO.

En el Servicio de ORL del Hospital San Juan de Dios (HSJD), el manejo estándar de un paciente con carcinoma escamoso de laringe transglótico consiste en el manejo quirúrgico del primario y del cuello en forma bilateral. Cuando se sospecha compromiso extracapsular de una adenopatía ( $>3$ $\mathrm{cm}$ ) se realiza un vaciamiento cervical radical; en aquellos casos en que se puede conservar alguna de las estructuras no ganglionares del vaciamiento radical clásico por su lejanía con la adenopatía, en la que se sospecha invasión extracapsular, se realiza un vaciamiento cervical radical modificado. $\mathrm{Si}$ las adenopatías son menores de $3 \mathrm{~cm}$ y no presentan invasión extracapsular, se realiza un vaciamiento cervical funcional.

Considerando los antecedentes aportados por la literatura, decidimos evaluar los resultados histopatológicos de los vaciamientos cervicales realizados en pacientes portadores de CTG Con estos datos pretendemos realizar una propuesta de manejo cervical.

\section{MATERIAL Y MÉTODO}

Se realizó un estudio retrospectivo, mediante la revisión de fichas de pacientes portadores de carcinoma escamoso de laringe, atendidos en el Servicio de ORL del HSJD entre enero de 1994 y diciembre de 2002. Se incluyeron en la revisión los pacientes con diagnóstico de carcinoma transglótico, definido como aquel tumor laríngeo, que en el informe de histopatología de las muestras tomadas por laringoscopía directa y/o en el análisis de la pieza operatoria de la laringectomía, demostrara el compromiso neoplásico de banda, ventrículo y cuerda vocal. Como adenopatía clínica se definió a aquella adenopatía de $1 \mathrm{~cm}$ o más, detectada clínicamente y confirmada por histopatología. La etapificación del tumor (TNM) fue reali- zada utilizando los criterios de la American Joint Committee on Cancer del año 2001 (AJCC). Se excluyeron los pacientes con antecedente de radioterapia cervical previa o cuyo motivo de consulta fue recidiva tumoral.

Todos los pacientes fueron manejados con intención curativa, mediante cirugía del primario y el cuello según compromiso tumoral.

Se evaluó la incidencia de metástasis clínica e histopatológica, la modalidad del vaciamiento cervical, el tipo de manejo quirúrgico del primario, diseminación ganglionar extracapsular, y la asociación entre edad, estadio del T, compromiso de línea media y riesgo de compromiso ganglionar.

$\boxminus$ análisis de los resultados se realizó mediante métodos descriptivos dado el reducido tamaño de la muestra.

\section{RESULTADOS}

En el periodo de estudio consultaron 83 pacientes con carcinoma laríngeo, 20 de los cuales cumplieron con los criterios de inclusión. La mediana de edad fue de 67 años, con un rango de 52 a 78 años. La distribución por sexo fue 17 (85\%) hombres y 3 (15\%) mujeres (Tabla 1).

La distribución según la etapa del T clínico fue: T2, cuatro $(20 \%)$, T3, trece $(65 \%)$ y T4, tres pacientes (15\%). Según hispatología la distribu-

Tabla 1. Características demográficas $(n=20)$

\begin{tabular}{|llc|}
\hline Sexo & Masculino & $17(85 \%)$ \\
& Femenino & $3(15 \%)$ \\
Edad & Rango & № \\
& $50-59$ & 3 \\
& $60-69$ & 11 \\
& $>70$ & 6 \\
Tabaquismo & $\mathrm{Si}$ & $17(85 \%)$ \\
& No & $3(15 \%)$ \\
\hline
\end{tabular}

Pacientes portadores de carcinoma transglótico incluidos en la muestra. 
ción fue T3, siete (35\%) y T4, trece pacientes (65\%) (Tabla 2).

日 manejo del primario consistió en laringectomía total en 19 pacientes y laringectomía casi total de Pearson en 1 paciente. En 16 pacientes no se detectaron adenopatías clínicas (80\%), realizándose en todos un vaciamiento ganglionar selectivo bilateral supraomohioideo (niveles II-III) o lateral (niveles II-III-IV), adicionando el nivel VI en aquellos casos con compromiso de subglotis. Cuatro pacientes $(20 \%)$ se presentaron con adenopatías clínicas, realizándose un vaciamiento ganglionar radical en dos de ellos (uno radical clásico y uno radical modificado), correspondiente a pacientes $\mathrm{N} 2$, y un vaciamiento funcional en los otros dos pacientes, correspondientes a N1. Los cuatro pacientes con adenopatías clínicas presentaron la enfermedad ganglionar ipsilateral al tumor (Tabla 3).

Se demostró metástasis ganglionar oculta en 2 de los 16 pacientes No (12,5\%) y en el cuello contralateral de uno de los pacientes $\mathrm{N}+$ clínico. Este último paciente no se considera en el total de las metástasis ganglionares ocultas por ser el cuello ipsilateral positivo. Si consideramos la distribución de metástasis ocultas por lateralidad del vaciamiento cervical, de las 16 disecciones cervicales ipsilaterales clínicamente negativas 2 $(12,5 \%)$ presentaron metástasis, y de los 20 vaciamientos contralaterales, todos clínicamente negativos, $1(5 \%)$ presentó metástasis (Tabla 4).

日 número promedio de ganglios de los vaciamientos ipsilaterales fue de 12 , con un rango de 2 a 41 . En los contralaterales el promedio fue de 9 con un rango de 2 a 19. Se solicitó, al departamento de anatomía patológica, una revisión de los ganglios comprometidos por metástasis $(n=25)$ en busca de diseminación extracapsular, sin observarse compromiso en ninguno de los ganglios analizados.

Las presencia de metástasis ganglionares histológicas $(n=6)$ según rango etáreo fue: rango de 50-59 años, 1 de 3 pacientes (33\%); 60-69 años, 3 de 11 pacientes (27\%) y en el rango de 70 79 años, 2 de 6 pacientes (33\%) (Figura 1). 日 compromiso ganglionar metastásico según Tfue: 1 paciente T3 (14\%) y 5 pacientes T4 (38\%) (Tabla 5). 日 compromiso de la línea media se evidenció
Tabla 2. Distribución por estadio $(\mathrm{n}=20)$

\begin{tabular}{|ccc|}
\hline Estadio TNM & Cínico & Histopatológico \\
\hline I & 0 & 0 \\
II & $4(20 \%)$ & $1(5 \%)$ \\
III & $10(50 \%)$ & $3(15 \%)$ \\
IV & $6(30 \%)$ & $16(80 \%)$ \\
\hline
\end{tabular}

Distribución de los pacientes por estadio TNM según hallazgos clínicos e histopatológicos.

Tabla 3. Distribución de pacientes según adenopatías clínicas e histopatológicas $y$ lateralidad $(n=20)$

\begin{tabular}{|lccc|}
\hline Pacientes N(+) & Ipsi & Contra & Total \\
\hline Cínico & 4 & 0 & $4 / 20(20 \%)$ \\
Histopatológico & 6 & $1^{*}$ & $6 / 20(30 \%)$ \\
\hline
\end{tabular}

* Paciente con adenopatíaipsilateral histopatológica Distribución de los pacientes de acuerdo a la presencia de adenopatías detectadas clínicamente e histopatológicamente, según lateralidad en relación al tumor.

Tabla 4. Distribución de metástasis ocultas por vaciamiento cervical ipsilateral $(n=16)$ y contralateral $(n=20)$

\begin{tabular}{|llrr|}
\hline & & \multicolumn{1}{c}{$\mathrm{pN}_{+}$} & \multicolumn{1}{c|}{$\%$} \\
\hline Ipsilateral N0 & $(\mathrm{n}=16)$ & 2 de 16 & $12,5 \%$ \\
Contralateral N0 $\quad(\mathrm{n}=20)$ & 1 de 20 & $5 \%$ \\
\hline
\end{tabular}

Distribución e incidencia de metástasis ocultas según la lateralidad del vaciamiento cervical No, en relación al tumor, en el cual fueron detectadas.

Tabla 5. Incidencia de metástasis por categoría T histopatológico pT ( $n=6)$

\begin{tabular}{|ccc|}
\hline pT1 & 0 & 0 \\
pT2 & 0 & $(0 / 1)$ \\
pT3 & $25 \%$ & $(1 / 4)$ \\
pT4 & $33 \%$ & $(5 / 15)$ \\
\hline
\end{tabular}

Incidencia de metástasis cervicales confirmadas por histopatología según la categoría del Thistopatológico. 


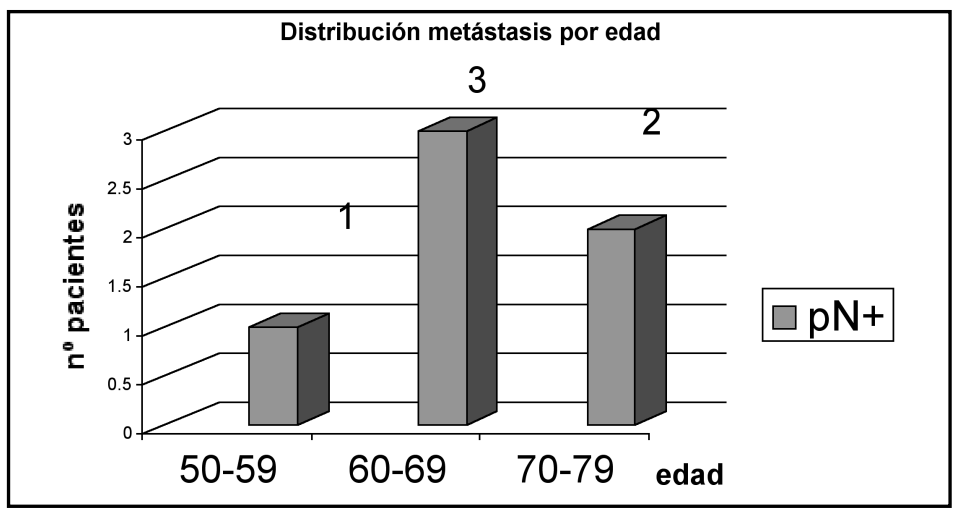

Figura 1. Distribución de metástasis en ganglios cervicales ganglionares según la edad del paciente.

en 18 pacientes (90\%), presentando adenopatías contralaterales sólo 1 de ellos.

\section{DISCUSIÓN}

日 correcto manejo, de los territorios de diseminación linfática cervical en los pacientes con carcinoma escamoso de cabeza y cuello es de gran importancia, considerando que la presencia de metástasis cervicales es el indicador más importante de recurrencia de la enfermedad y de sobrevida. Existe consenso respecto a considerar el manejo quirúrgico regional en pacientes con adenopatías cervicales mediante disección radical (clásica o modificada) o funcional como la mejor alternativa terapéutica en aquellos pacientes en los cuales la modalidad de manejo del tumor primario será quirúrgica, conducta terapéutica que corresponde a la seguida en nuestro hospital. En pacientes NO la conducta está en discusión. La presencia de metástasis ganglionar oculta en algunas localizaciones tumorales laríngeas supera el 30\%. Es lógico pensar que estas metástasis llegarán a ser clínicamente aparentes en los cuellos no tratados, como se aprecia en la incidencia de cuellos histológicamente positivos y su correlación con el porcentaje de conversión de los cuellos no tratados ${ }^{18}$. Por otro lado, la sensibilidad de los métodos clínicos, inclu- yendo tomografía computada y resonancia nuclear magnética, llega al 80\%-85\% ${ }^{19,20}$ situación que acarrea un riesgo considerable de falsos negativos y de falla en el manejo ganglionar. Varios estudios que revelan la presencia de antecedentes a favor de una conducta quirúrgica, por sobre la observación, a la espera de la aparición de metástasis cervical están basados en la falla del tratamiento del cuello en el $23 \%$ a $38 \%$ de los casos al presentarse adenopatías posteriormente ${ }^{21}$. Dado los antecedentes anteriores, diversos autores sugieren realizar un manejo quirúrgico ganglionar en localizaciones tumorales con un riesgo de metástasis ganglionar oculta sobre el 20\% $22-25$, situación descrita en el carcinoma transglótico.

Myers ${ }^{17}$, contrariamente a lo descrito previamente, describe una baja incidencia de estas metástasis en el carcinoma transglótico (12\%), situación descrita también en otros estudios ${ }^{26}$. Nuestro trabajo demostró la presencia de metástasis en 2 pacientes clínicamente negativos (12,5\%) y además en el cuello contralateral al tumor de un paciente con metástasis clínica en el cuello ipsilateral. Estos hallazgos concuerdan con lo descrito por Myers y otros autores, y sugerirían no realizar un vaciamiento cervical en pacientes NO clínico, por ser la incidencia de metástasis ganglionar oculta menor al $20 \%$; esto es particularmente válido en el cuello contralateral, donde la 
incidencia fue de 5\%. Sin embargo, existen elementos que favorecen el adoptar una conducta quirúrgica a nivel ganglionar, al menos ipsilateral, tales como el tratarse de un paciente en que la resolución del primario será preferentemente quirúrgica y por el hecho que el vaciamiento ganglionar funcional no agrega una morbilidad adicional significativa. No obstante, es válido plantearse la inconveniencia del manejo quirúrgico en carcinoma transglótico N0, aun cuando son necesarios más estudios que permitan demostrar la incidencia exacta de metástasis oculta.

日 compromiso ganglionar extracapsular (EC) es un factor de mal pronóstico, aumentando el riesgo de falla en el control regional 27,28 . Es menos frecuente en los ganglios de menor tamaño (23\% en $<1 \mathrm{~cm})$. Sin embargo, producto de la progresión de la enfermedad, ganglios sin compromiso EC pueden llegar a desarrollar, a futuro, una diseminación de tal magnitud que haga a la metástasis inoperable. En nuestro estudio no se observó compromiso extracapsular en ninguna de las muestras analizadas. Se solicitó una nueva revisión histopatológica de los ganglios metastásicos, estudiándose un corte de todo el ganglio (lo disponible), situación que no modificó el resultado inicial. 日 estudio de un solo corte puede no ser representativo de lo que ocurre en toda la estructura nodal, por lo cual no se puede asegurar la ausencia de compromiso en otras áreas de la cápsula ganglionar.

Llama la atención el bajo número de ganglios informados por histopatología, en promedio 12 ganglios en los vaciamientos ipsilaterales y 9 ganglios en los contralaterales. Diversos autores describen un promedio mayor de ganglios disecados en disecciones cervicales selectivas $\mathrm{SHO}$ y laterales como Hosal y col${ }^{29}$ (23 ganglios), Coskun y col$^{30}$ (31,14 ganglios) y el Grupo de Estudio de Cáncer de Cabeza y Quello Brasileño ${ }^{31}$ (30 ganglios). Además, en estas series, como en nuestro trabajo, se presenta un amplio rango en el número de ganglios disecados, pese a haber sido procedimientos realizados por un cirujano de experiencia y en forma estandarizada, situación que podría explicarse por el análisis realizado a la pieza operatoria por histopatología. Así, el bajo promedio de ganglios descritos en nuestro estudio implica un sesgo importante, al momento de obtener resultados comparativos con otras publicaciones.

Entre los diversos factores de riesgo para desarrollar metástasis ganglionar se ha descrito la edad avanzada, etapa tumoral avanzada y el compromiso de la línea media. En nuestro estudio la presencia de metástasis se distribuyó en forma homogénea entre los distintos grupos etáreos. La etapa tumoral avanzada se relaciona con una mayor presencia de ganglios metastásicos como lo revela el estudio presentado por Tomic y $\mathrm{col}^{32}$, describiendo en T1 ausencia de metástasis ganglionar y en T4 44,2\%. En nuestra serie, según T histológico, todos los casos eran de etapas avanzadas (T3, 35\% y T4, 65\%) y el compromiso ganglionar metastásico correspondió a T3 14\% y T4 33\%, mostrando un predominio por etapa del T más avanzada. Por último, observamos que el compromiso de la línea media no se asoció a una mayor incidencia de metástasis bilateral.

日 análisis de los datos anteriormente descritos, particularmente en lo que respecta a la edad y compromiso de la línea media, y su relación con la presencia de metástasis ganglionares cervicales parecen demostrar que no existe una relación directa, sin embargo, debido al bajo número de casos de la serie no es posible obtener conclusiones al respecto.

\section{CONCLUSIONES}

1. Se encontraron adenopatías clínicas en el $20 \%$ de los pacientes.

2. 日 $12,5 \%$ de los pacientes NO tuvieron metástasis ocultas.

3. 日 $12,5 \%$ en los vaciamientos ipsilaterales $(n=16)$ y el $5 \%$ en los vaciamientos contralaterales $(n=20)$, clínicamente negativos presentaron metástasis ocultas.

4. Considerando la baja incidencia de metástasis ocultas $(<20 \%)$ los carcinomas de ubicación transglótica NO podrían no beneficiarse de un vaciamiento cervical funcional contralateral, ya 
que su frecuencia de adenopatías ocultas es baja (5\%). Con respecto al vaciamiento ipsilateral, según nuestros resultados tampoco se justificaría (12,5\% de metástasis ocultas).

5. La edad y compromiso de la línea media parecieran no estar relacionados a una mayor incidencia de metástasis ganglionar cervical, no obstante, debido al bajo número de casos de la serie no es posible obtener conclusiones al respecto.

6. Esta conducta expectante deberá ser evaluada en el contexto de un protocolo prospectivo.

\section{BIBLIOGRAFÍA}

1. KOUFMAN J, BURKE, A. The etiology and pathogenesis of laryngeal carcinoma. Atolaryngologic clinics 1997; 30: 11-9.

2. SHWARTZ MR. Pathological of Laryngeal Tumors. In: Comprehensive management of head and neck tumors. Philadelphia, W.B. Saunders Company 1999; 950-78.

3. Contrgras JM, NikLITSCheK E, Paredes A, MaAsS J. Cáncer laríngeo-hipofaríngeo. Evaluación en el Hospital San Juan de Dios. Rev Otorrinlaringol Or Cabeza y Ouello 2001; 61: 19-30.

4. Pradenas M, Vallejos M, Rahal M, GisterNAS A. Cáncer de laringe e hipofaringe. Experiencia del Hospital Barros Luco Trudeau 1990-2001. Rev Otorrinlaringol Cir Cabeza y Quello 2004; 64: 13-20.

5. SOHULler DE, Mc GUIRT WF, MOCABE BF, YOUNG D. The prognostic significance of metastasis cervical lymph nodes. Laryngoscope 1980; 90: 557-70.

6. Cachin Y, Sancho-Garnierh, Micheau C, MaRANDAS P. Nodal metastasis from carcinoma de oropharynx. Aolaryngol Cin North Am 1979; 12: $145-54$.

7. Mamelle G, Pampurix J, LubOnSKi B et al. Lymph node prognostic factors in head and neck squamous cell carcinomas. Am J Surg 1994; 168: 494-8.

8. CANDEA $\mathrm{HK}$, SHaH J, JaQues DP, SHaH JP. Patterns of cervical node metastasis from squamous carcinoma of the larynx. Arch Otolarynglo Head Neck Surg 1990; 116: 432-435.

9. VANDENBROUCK C, SANCHO-GARNIIR H, OHASSAG NED, SARAVANE D, CAGHIN Y, Micheau C. Bective versus therapeutic radical neck dissection in epidermoid carcinoma of the oral cavity: results of a randomized clinical trial. Cancer 1980; 46: 386-90.

10. BOOCA E PIGNATARO O. A conservation techinique in radical neck dissection. Ann Otol Rhinol Laryngol. 1967; 76: 975-87.

11. W'BR PC, JOHNSON JT, MYES $\mathrm{EN}$. The impact de bilateral neck dissection on pattern of recurrence and survival in supraglottic carcinoma. Arch Otolaryngol Head Neck Surg 1994; 120 : 703-6.

12. KIRCHNER JA, CORNOG JL, HOLMES RE Transglottic cancer. Arch Aolaryngol. 1974; 99: 247-51.

13. BILleR HF, LAWSON W. Parcial laryngectomy for transglottic cancer. Ann Otol Rhinol Laryngol 1984; 93: 297-300.

14. WeSH LW, SCHUNKE M, RIZZO TA. Laryngeal spaces and lymphatics: currrent anatomic concepts. Ann Otol Rhinol Laryngol 1983; 92: 19-31.

15. Werner LA, SCHUNKE M, RUdert H, TILLMAN B. Description and clinical importance of the lymphatics of the vocal fold. Otolaryngol Head and Neck Surgery 1990; 102: 13-9.

16. W且S LW, WeSSHJJ, RIZZOTA. Internal anatomy of the larynx and the spread of cancer. Ann Otol Rhinol Laryngol 1989; 98: 228-34.

17. MYERS 日N, FAGAN JJ. Management of the neck in cancer of the laynx. Ann Otol Rhinol Laryngol 1999; 108: 828-32.

18. SNOW GB, PATE P, LEMANS CR, TIWARI R. Management of cervical lymph nodes in patients with head and neck cancer. Eur Arch Otorhinolaryngol 1992; 249: 187-94.

19. Van Den Brekel MMM, Castelijins JA, Snow GB. Diagnostic evaluation of neck. Otolaryngol Cin North Am 1998; 31: 601-19

20. Kau RJ, Alexiou C, Stimmer h, Arnold W. Diagnostic procedures for detection of lymph node metastasis in cancer of the larynx. ORL $J$ Otorhinolaryngol Relat Spec 2000; 62: 199203. 
21. CACHIN Y. Management of cervical nodes in head and neck cancer. In: Rhys Evans PH, Robin PE, Fielding JWL, eds. Head and neck cancer. London, England: Castle House Publications Ltd. 1983: 168-177.

22. WeSS MH, HARRISON LB, ISAACS RS. Use of decision analysisin planning a management strategy for the stage N0 neck. Arch Otolarynglo Head Neck Surg 1994; 120: 699-702.

23. YUeN AP, HO CM, WE WI, LAM LK. Prognosis of recurrent laryngeal carcinoma after laryngectomy. Head Neck 1995; 17: 526-30.

24. KOWALSKI LP, BAGIETTO R, LARA JR, SANTOS RL, SILVA JF, MAGRIN J. Prognostic significance of the distribution of neck node metas- tasis fron oral carcinoma. Head Neck 2000; 22: 207-14.

25. OGURA JH, BiLler HF, Wette R. Bective neck dissection for pharingeal and laryngeal cancer: an evaluation. Ann Otol Rhinol Laryngol 1971; 80: 646-51.

26. MitTAL B, MaRKS JE, OGURA JH. Transglottic carcinoma. Cancer 1984; 53: 151-61.

27. SNOW GB, ANNYAS AA, VAN SLOOIEN EA, BARTE LINK H, HART AA. Prognostic factors of neck node metastasis. Cin Aolaryngol 1982; 7: 185-92.

28. JOHNSON JT, BARNES 日, MYERS EN, SCHRAMM VL, BOROAHOVIZ D, SIGLr BA. The extracapsular spread of tumors in cervical node metastases. Arch Otolaryngol 1981; 107: 725-9. 